\title{
Theoretical Studies of Bounce in Rod-Type Microswitches
}

\author{
E.S. Lyuminarskaya ${ }^{1,}{ }^{*}$ \\ ${ }^{1}$ Bauman Moscow State Technical University, Block 1, Bldg. 5, 2nd Baumanskaya Str., Moscow, \\ Russian Federation, 105005
}

\begin{abstract}
To improve reliability of microswitches, it is necessary to carry out theoretical studies of the switching process both at the moment of switching and at the moment of bouncing. The article presents a dynamic model of microswitches with elastic rod elements, which allows at the design stage to evaluate such basic performance characteristics as response time and contact bounce time. The proposed technique is based on representation of the solution to the differential equation of vibrations of an elastic element in the form of a linear combination of eigenforms and the application of the Bubnov-Galerkin method. Theoretical studies of bounce in a rod microswitch are carried out. It is shown that breakdown precursors of the spacer spring are an increase in the switching time and the time of the first separation in case of bouncing.
\end{abstract}

\section{Introduction}

Failures in low-level contacts of rod-type microswitches mainly appear due to breakage of an elastic element or a spacer spring, sticking of a moving contact, contact bounce during switching, a sudden change in the contact pressing force, contamination of contact surfaces, etc. To increase reliability of microswitches, it is necessary at the design stage to carry out theoretical studies of the response time, taking into account contact bounce.

To create reliable miniature switches, it is necessary to develop mathematical models that take into account contact bounce during switching, and use them to identify the main causes and precursors of failures and breakdowns at the design stage.

\section{Problem formulation}

When elastic contacts are closed for a short time (less than $1 \mathrm{~ms}$ ), the contacts are repeatedly closed and opened (bounce). The bounce can cause malfunctions of microcircuits and lead to damage of electronic equipment. Therefore, when choosing the parameters of microswitches, it is necessary to calculate the response time and bounce, to obtain their dependence on the elastic parameters of the system. These dependencies will help to predict possible switching failures during operation.

\footnotetext{
* Corresponding author: lyuminarskaja.caterina@yandex.ru
} 
The existing methods for calculating vibrations of contact systems with elastic rod elements and elastic constraints are based on the use of the finite element method [1-5]. In contact systems, elastic restraints have a high contact stiffness. When the elastic element hits the restraint, high-frequency vibration modes are excited in it, which must be taken into account when studying contact bounce. Therefore, in this work, the Bubnov-Galerkin method is used to calculate the vibrations of an elastic system. The eigenforms [6], [7], [8] of an elastic element with a movable contact are used as basis functions. The use of this method can significantly reduce the counting time. The proposed technique makes it possible to determine the movement of the elastic element both at the moment of switching and at the moment of bouncing.

The purpose of this work is to develop a methodology for the dynamic analysis of bounce in microswitches with elastic rod elements and theoretical studies of contact bounce in a rod-type microswitch.

\section{Mathematical model}

The design diagram of a microswitch with an elastic rod element is shown in Figure 1. The speed of microswitches is mainly provided by such a selection of parameters when all equilibrium positions of an elastic element with a spacer spring without support on fixed contacts are unstable. The instability of the equilibrium positions is provided by small angles of inclination of spacer spring $\alpha$ (see Fig. 1). In this case, even with small displacements of the cross-sections along the y-axis, the longitudinal component of the force from the side of spacer spring $\vec{S}_{x}$ will have a significant effect on the elastic element movement.

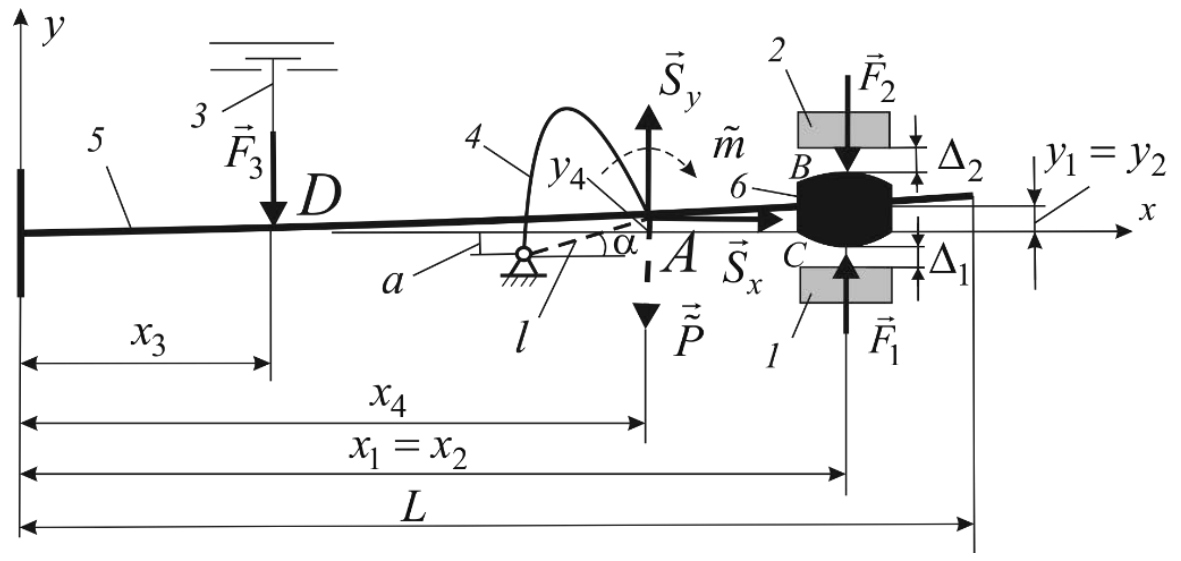

Fig. 1. Design diagram of a rod microswitch:

1,2 - fixed contacts; 3 - pusher; 4 - spacer spring; 5 - elastic element; 6 - movable contact boss

The longitudinal component of the force from the side of spacer spring $\vec{S}_{x}$ is approximately replaced by lateral force $\tilde{P}$ and bending moment $\tilde{m}$. With this replacement, the nonlinear dependence of the internal bending moment on the longitudinal coordinate $M_{S_{x}}(x)=S_{x} \cdot\left(y-y_{4}\right)$ is replaced by a linear one $\tilde{M}_{S_{x}}(x)=-\tilde{P} \cdot\left(x_{4}-x\right)-\tilde{m}$ in section $\left(0, x_{4}\right)$. Coefficients $\tilde{P}$ and $\tilde{m}$ are determined by the least squares method from the condition 


$$
\int_{0}^{x_{4}}\left(M_{S_{x}}(x)-\tilde{M}_{S_{x}}(x)\right)^{2} d x=\min _{\tilde{P}, \tilde{m}},
$$

After transformations, the formula is obtained to determine $\tilde{P}$ and $\tilde{m}$

$$
\begin{aligned}
& \tilde{P}=6 S_{x}\left(2 x_{4}^{-3} \cdot \int_{0}^{x 4} x y d x-x_{4}^{-2} \cdot \int_{0}^{x_{4}} y d x\right), \\
& \tilde{m}=S_{x}\left(y_{4}+2 x_{4}^{-1} \cdot \int_{0}^{x_{4}} y d x-6 x_{4}^{-2} \cdot \int_{0}^{x 4} x y d x\right) .
\end{aligned}
$$

The differential equation of the beam movement during bending, taking into account the internal friction losses, has the following form [7]

$$
m_{0} \frac{\partial^{2} y}{\partial t^{2}}+\frac{\partial^{2}}{\partial x^{2}}\left[E \cdot J \cdot \frac{\partial^{2}}{\partial x^{2}}\left(y+\beta \cdot \frac{\partial y}{\partial t}\right)\right]=q(x, t),
$$

where $y(t, x)$ - beam displacement function; $m_{0}$ - running weight; $E$ - modulus of elasticity of the first type; $J$ - cross-sectional moment of inertia; $\beta$ - dissipation parameter; $q(x, t)$ - intensity of the distributed force acting on the elastic element.

Concentrated forces act on the beam (see Fig. 2). Therefore, intensity $q(x, t)$ is represented by a linear combination of the Dirac delta functions $\delta\left(x-x_{i}\right)$ :

$$
\begin{array}{r}
q(x, t)=S_{y} \cdot \delta\left(x-x_{4}\right)-\tilde{P} \cdot \delta\left(x-x_{4}\right)-\tilde{m} \cdot \delta^{\prime}\left(x-x_{4}\right)+F_{1}(t) \cdot \delta\left(x-x_{1}\right)+ \\
-F_{2}(t) \cdot \delta\left(x-x_{2}\right)-F_{3}(t) \cdot \delta\left(x-x_{3}\right)-m \cdot \frac{\partial^{2} y}{\partial t^{2}} \cdot \delta\left(x-x_{2}\right),
\end{array}
$$

where $m$ - the boss mass.

The vertical component of the force from the spacer spring is determined from the expression

$$
S_{y}=S \cdot \sin (\alpha)=d \cdot\left(y_{4}+a\right),
$$

where $d=\frac{k\left(l_{0}-l\right)}{l} ; k$ - stiffness coefficient of the spacer spring; $l_{0}, l-$ length of undeformed and deformed spacer spring.

The solution to equation (1) is represented by a linear combination of the eigenforms of elastic element 5 with boss 6 (see Fig. 1)

$$
y(t, x)=\sum_{j=1}^{N} \xi_{j}(t) \cdot \tilde{y}_{j}(x),
$$

where $\tilde{y}_{j}(x)$ - kinetic energy normalized eigenforms of an elastic element with a boss; $\xi_{j}(t)-$ unknown functions.

After substitution of expressions (2) and (3) into equation (1), a partial differential equation is obtained, in which functions $\xi_{j}(t)$ are unknown. This differential equation is scalarly multiplied by normalized eigenforms $\tilde{y}_{j}(x)$. The scalar product of two different eigenforms is equal to zero, therefore all differential equations for determining functions $\xi_{j}(t)$ are independent. Consequently, to determine functions $\xi_{j}(t)$, it is necessary to solve homogeneous differential equations of the second order

$$
\ddot{\xi}_{i}(t)+2 n_{i} \cdot \dot{\xi}_{i}(t)+\omega_{i}^{2} \cdot \xi_{i}(t)=d \cdot\left(y\left(t, x_{4}\right)+a\right) \cdot \tilde{y}_{i}\left(x_{4}\right)+
$$


$F_{1}(t) \tilde{y}_{i}\left(x_{1}\right)-F_{2}(t) \tilde{y}_{i}\left(x_{2}\right)-F_{3}(t) \tilde{y}_{i}\left(x_{3}\right)-\tilde{P} \cdot \tilde{y}_{i}\left(x_{4}\right)+\tilde{m} \cdot \tilde{y}_{i}^{\prime}\left(x_{4}\right)$,

where $i=1, \ldots, N ; \omega_{i}$ - natural frequencies of an elastic element with a boss; $n_{i}-$ damping coefficient of the i-th eigenform; $\tilde{y}_{i}^{\prime}=\partial \tilde{y}_{i} / \partial x$.

Dependences of reaction forces $F_{1}, F_{2}$, and $F_{3}(t)$ on the approach of contact surfaces are determined by the Hertz formula.

The approach of contact surfaces depends on the movement of the points of elastic element $y_{1}, y_{2}, y_{3}$ and initial clearances $\Delta_{10}, \Delta_{20}, \Delta_{30}$

$\Delta_{1}(t)=\Delta_{10}+y\left(t, x_{1}\right), \Delta_{2}(t)=\Delta_{20}-y\left(t, x_{2}\right), \Delta_{3}(t)=\Delta_{30}-y\left(t, x_{3}\right)$.

To solve equations (3) - (5), the integration section $\left(0, t_{k}\right)$ is divided into $M$ equal intervals. On each interval, the variable values $d, y\left(t, x_{4}\right), F_{1}, F_{2}, F_{3}, \tilde{P}, \tilde{m}$ are considered constant and are taken equal to the average values at the integration step.

At the initial moment of time, the pusher is located in the extreme upper position and abuts against the restraint, and the elastic element with the boss is in equilibrium. The calculation is carried out at a constant speed of the pusher, which moves from the extreme upper to the extreme lower position.

At the initial moment of time, derivatives $\dot{\xi}_{i 0}=0$ and functions $\xi_{i 0}$ are determined from equations (4), where all derivatives with respect to time are equated to zero. The values of the quantities included in the right-hand side of the differential equations (4) are determined from the calculation of the equilibrium of the elastic element.

To solve the system of equations under consideration, the method of introducing restoring forces is used [10] to calculate elastic systems with one-way constraints.

The microswitch works as follows. In the free state, pusher 3 is in the extreme upper position and abuts against the restraint. Movable contact 6 is pressed against fixed contact 2 with a certain pressing force. When the pusher moves down (forward stroke), the pressing force of contacts 2, 6 decreases. At the moment when force $F_{2}$ becomes equal to zero, the system, consisting of elastic element 5 , spacer spring 4 and boss 6 , will be in a position of unstable equilibrium. With a further (arbitrarily small) increase in the control force, the boss jumps to the lower position, i.e. an instantaneous action mechanism (clap) is triggered. After jumping, the pusher can move to the lower limit position, determined by pusher stop 8.

\section{Research results}

For a theoretical study, a microswitch with the following parameters was chosen (see Fig. 1): $x_{1}=x_{2}=10.5 \mathrm{~mm} ; x_{3}=4.8 \mathrm{~mm} ; x_{4}=9 \mathrm{~mm} ; L=12 \mathrm{~mm}$; the stiffness coefficient of the spacer spring (4) $k=3 . .7 \frac{N}{\mathrm{~mm}} ; a=0$; the width and thickness of the elastic element $5 \mathrm{~b}=4.2 \mathrm{~mm}, h=0.1 \mathrm{~mm}$; the gaps between the movable and fixed contacts with an undeformed elastic element $5 \Delta_{1}=-0.15 \mathrm{~mm}, \Delta_{2}=0.45 \mathrm{~mm}$; the gap between the pusher, pressed against the upper restraint, and undeformed elastic element (5) $\Delta_{3}=$ $0.197 \mathrm{~mm}$; pusher movement speed $v_{3}=10 \frac{\mathrm{m}}{\mathrm{s}}$; dissipation parameter $\beta=2 \cdot 10^{-6} \mathrm{~s}$. 


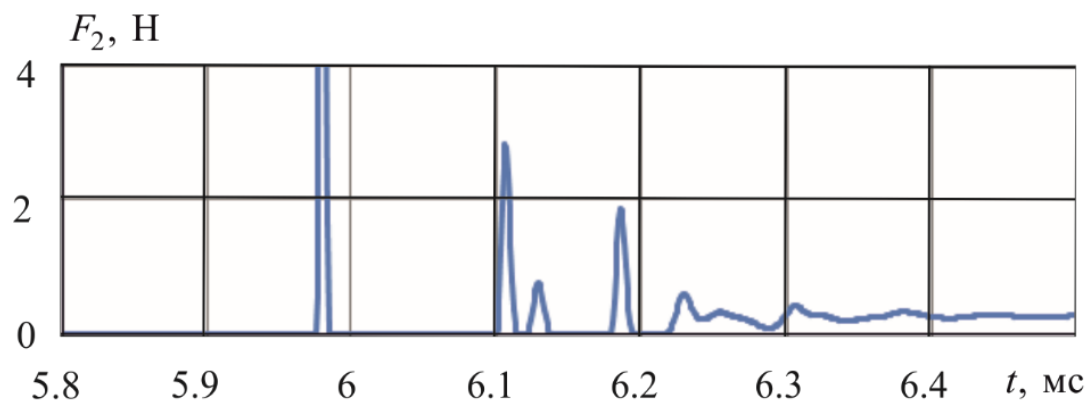

Fig. 2. Changing of contact pressing forces during switching process

Figure 2 shows a graph of the change in the contact pressing force $F_{2}$ at the moment of switching with the stiffness of the spacer spring7 $\mathrm{N} / \mathrm{mm}$ The contact pressing force in the steady state is $0.64 \mathrm{~N}$. It can be seen from the figure that contact bounce occurs during commutation. At the bounce moment, there are four detachments. The time of the first detachment is $0.12 \mathrm{~ms}$, the second $-0.005 \mathrm{~ms}$, the third $-0.041 \mathrm{~ms}$, and the fourth -0.021 ms. Calculations with different stiffness of the spacer spring showed that with bounce, two or three large detachments $\left(t_{\mathrm{o}}>0.01 \mathrm{~ms}\right)$ are mainly manifested.

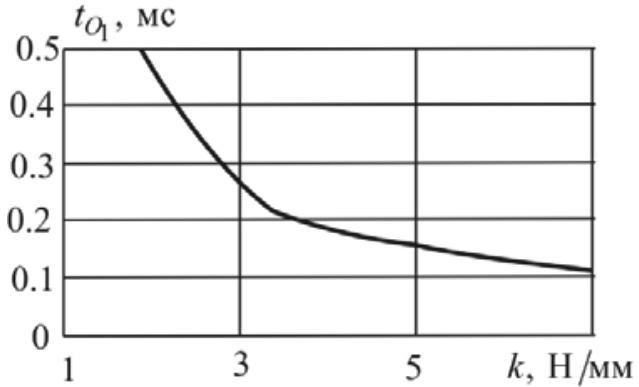

Fig. 3. Time of first detachment at bounce

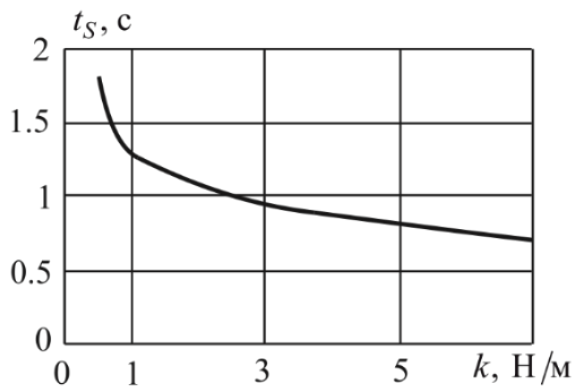

Fig. 4. Switching time

It can be seen from the presented dependence that at the first interaction, the contact force increases sharply. The maximum force is more than 10 times the nominal contact force. The time of the first encounter of the contacts is less than $7 \mu \mathrm{s}$. During this time, large deformations and stresses do not have time to appear in the elastic element of the microswitch.

One of the reasons for the failure of microswitches may be breakdown of spacer spring 4. To identify the precursors of this failure, calculations of commutations were carried out at different stiffness of the spacer spring $k$. Figure 3 shows the dependence of time of the first maximum detachment of contacts with bounce $t_{O_{1}}$ on the specified stiffness. Decreasing the stiffness from $7 \mathrm{H} / \mathrm{MM}$ to $3 \mathrm{H} / \mathrm{MM}$ increases $t_{O_{1}}$ by 2.3 times. A further decrease in stiffness leads to a more rapid increase in the time of the first detachment.

Figure 4 shows the dependence of the switching time on the stiffness of the spacer spring. The switching time $t_{s}$ is the time between disconnection of the boss with the upper contact and its first encounter with the lower contact. Decreasing the stiffness from $7 \mathrm{H} / \mathrm{Mm}$ to $1 \mathrm{H} / \mathrm{Mm}$ increases the switching time by 1.9 times. With a further decrease in the stiffness of the spacer spring, the switching time increases sharply.

Therefore, the precursors of the breakage of the spacer spring are an increase in the switching time and the time of the first detachment in case of bouncing. 


\section{Conclusion}

1. A mathematical model of microswitches with elastic rod elements is considered, which makes it possible to study their operation in the phases of switching and contact bouncing.

2. Computational studies have shown that with contact bounce, 3-4 detachments occur with a detachment time of more than $10 \mu \mathrm{s}$.

3. The calculations showed that the forerunners of failures due to breakage of the spacer spring are an increase in the switching time and the time of the first detachment of contacts during bounce.

\section{References}

1. Alfutov N.A. Basics of calculation for the stability of elastic systems / N.A. Alfutov. M.: Mechanical Engineering, 1978. -311 p.

2. Bazhenov V.A. Stability and oscillations of deformable systems with one-sided connections / V.A. Bazhenov, E.A. Gotsulyak, G.S. Kondakov, A.I. Ogloblya. - Kiev: High school. Head Publishing House, 1989. - 399 p.

3. Kokhmanyuk S.S. Dynamics of structures under the influence of short-term loads/ Kokhmanyuk S.S., Dmitriev A.S., G.A. Sheludko and others - Kiev: Naukova Dumka, 1989. - 304 p.

4. Contact systems / V.V. Ostashavichyus, B.V. Rudgalvis, V.L. Ragulskine, B.P. Bakshis. - L.: Mashinostroenie, 1987. - 279 p.

5. Ulanov A.V. Mathematical models of dynamics, algorithms and informationmeasuring means of vibroacoustic diagnostics and non-destructive testing of contact devices of aviation equipment: diss. Cand. Tech. Sciences: 05.11.16 and 05.11.13 / A.V. Ulanov. - Izhevsk.: RSL, 2006. - 178 p.

6. Vibration in technology: a handbook: In 6 volumes. Vol. 6: Protection against vibration and shock / ed. by K.V. Frolov. - 2nd ed., rev. and add. - M.: Mashinostroenie, 1995. - $456 \mathrm{p}$.

7. Vibration in technology: a handbook: In 6 volumes. Vol. 1: Oscillations of linear systems / ed. by V.V. Bolotin. - M.: Mashinostroenie, 1978. - 352 p.

8. Kollats L. Eigenvalue problems. - M.: Science. Ch. ed. Phys.-mat. lit., 1968. - p. 504.

9. Lyuminarskaya E.S., Lyuminarskiy I.E. Theoretical studies of precursors of failures in rod-type microswitches. Fundamental and applied problems of engineering and technology, 2017, No. 2-4 (324), pp. 25-33.

10. Luminarskiy I.E., Luminarskiy S.E. Method for calculating linear systems bounded by one-way connections under static loading. Vestnik MGTU named after N.E. Bauman. 'Mechanical Engineering' Series. - 2009. - No. 2. - pp. 84-90. 